\title{
A simple setup for episcopic microtomy and a digital image processing workflow to acquire high-quality volume data and 3D surface models of small vertebrates
}

\author{
Authors: Karolin Engelkes ${ }^{1}$, Frank Friedrich ${ }^{2}$, Jörg U. Hammel ${ }^{3,4}$, Alexander Haas ${ }^{1}$ \\ ${ }^{1}$ Center of Natural History (CeNak), Universität Hamburg \\ Martin-Luther-King-Platz 3 \\ 20146 Hamburg \\ Germany \\ ${ }^{2}$ Biocenter Grindel, Universität Hamburg \\ Martin-Luther-King-Platz 3 \\ 20146 Hamburg \\ Germany \\ ${ }^{3}$ Institute of Materials Research, Helmholtz-Zentrum Geesthacht \\ Max-Planck-Straße 1 \\ 21502 Geesthacht \\ Germany \\ ${ }^{4}$ Institut für Spezielle Zoologie und Evolutionsbiologie mit Phyletischem Museum, Friedrich- \\ Schiller-Universität Jena \\ Erbertstr. 1 \\ 07743 Jena \\ Germany \\ *Corresponding author: Karolin Engelkes \\ E-mail: karolin.engelkes@uni-hamburg.de \\ Telephone: +49 40 42838-4654
}

\section{Acknowledgements}

We thank Anna-Maria Vogt and Lena Schwinger for skillful help with the lab-work. We also thank the students who have worked with episcopic microtomy data in our class or during their bachelor projects and helped gaining experiences with the workflow, in particular Stine Griep, Lisa Malina Hahne, Vivienne-Christine Ahrens, Juliana Stahl, and Mehria Sedik. We also thank the anonymous reviewers for their helpful comments on the manuscript. 


\begin{abstract}
The use of volume data and digital three-dimensional (3D) surface models in biology has increased quickly and steadily. Various methods are available to acquire 3D data, among them episcopic imaging techniques. Based on the episcopic microscopy with on-block staining protocol of Weninger et al. (1998), we describe a simple and versatile setup for episcopic microtomy. It is composed of a consumer DSLR digital camera combined with standard histology equipment. The workflow of block surface staining and imaging, image processing, stack alignment, surface generation (including a custom Amira ${ }^{\circledR}$ macro), and 3D model editing is described in detail. For our sample specimen (Alytes obstetricans; Amphibia: Anura) we obtained images with a pixel size of $5.67 \times 5.67 \mu \mathrm{m}^{2}$. The generated image stacks allowed distinguishing different tissues and were well-suited for creating a 3D surface model. We analyzed the alignment quality achieved by various selections of specimen and fiducial marker spots. The fiducial spots had a significant positive effect on the alignment quality with the best alignment having a maximum mean alignment error of about $44.7 \mu \mathrm{m}$. We further tested the APS-C camera with combinations of macro lens, extension tube or teleconverter. The macro lens and extension tube yielded the smallest pixel size of $2.53 \times 2.53 \mu \mathrm{m}^{2}$. Considering data quality and resolution, and depending on object sizes and research goals, DSLR captured episcopic microtomy can be an alternative to other techniques, such as traditional histological sectioning or micro-computed tomography.
\end{abstract}

Keywords: block surface imaging; on-block staining; alignment; volume data; surface model 


\section{Introduction}

The use of digital three-dimensional (3D) volume representations and surface models for analysis and visualization increased substantially within the last two decades (e.g., Joschko et al. 1991; Haas 2001; Golding et al. 2009; Staedler et al. 2013; Lautenschlager et al. 2014; Gan et al. 2016; Heiss et al . 2016; Henne et al. 2017). 3D surface models have also been used increasingly in geometric morphometrics (e.g., Gunz et al. 2012; Piras et al. 2015; Werneburg et al. 2015), X-ray Reconstruction of Moving Morphology (e.g., Brainerd et al. 2010; Griep et al. 2013), multibody dynamic analyses and musculoskeletal modeling (e.g., Kargo and Rome 2002; Curtis et al. 2010; Charles et al. 2016), finite element analyses (e.g., Cox et al. 2011; Kleinteich et al. 2012; Fortuny et al. 2015), and computational fluid dynamics (e.g., Hammel et al. 2013; Rahman et al. 2015; also see Davies et al. 2017 and references therein).

In vertebrate morphology, arguably one of the first procedures to generate 3D models of biological structures was introduced by Born $(1876 ; 1883)$. He used stacked wax plates, cut out according to histological sections projected on them via a prism, to build a physical, enlarged 3D representation of amphibian nasal structures. Later progress in computer technology allowed creating digital 3D models of biologically relevant structures by surface scanning, by photogrammetry, or based on image stacks and volume data. Methods of volume data acquisition include micro-computed tomography $(\mu \mathrm{CT})$, magnetic resonance imaging (MRI), confocal laser-scanning microscopy (CLSM), or digitally stacked serial images of a specimen. A review of these and related techniques and the pertinent literature has been presented by Geyer et al. (2009). For the stacked image approach, digitized histological serial sections (e.g., Handschuh et al. 2010) and episcopic 3D imaging methods (i.e., methods that generate volume data by repetitively digitizing the cut surface of a histologically embedded specimen during its sectioning; also see Geyer et al. 2009) have been used.

Weninger et al. (1998) described an episcopic 3D imaging method to acquire highresolution volume data. They mounted a block with an embedded specimen on a microtome for sectioning and combined in situ staining of the cut surface (Hegre and Brashear 1946, 1947), with computer-assisted block surface digitizing and image processing techniques (Laan et al. 1989; Odgaard et al. 1990). Unlike traditional histological sections on slides, the main advantages of the episcopic imaging are that the resulting image stacks are gapless, rapidly generated, not affected by geometric distortions, and inherently aligned such that the natural outline of the specimen is preserved. Weninger et al. (1998) produced two stacks of grayscale images, one stack of unstained and one of stained block surfaces images, respectively. 
Due to the precise congruence of the cross section images, automatic image subtraction between stained and unstained images was possible and removed shining-through background structures (Weninger et al. 1998; fig. 1). They used the resulting stack of subtracted images for the generation of a surface model. In their setup with highly precise image capture position, post-sectioning alignment was unnecessary. If choosing digitized histological sections on slides (without positional precision in image capture) to create volume data, however, image alignment is an obligatory, yet an error-prone and time-consuming step in the preparation of volume data sets. Such alignment can result in unnatural shapes; for example, wrongly straightened curved structures (the so-called "banana" problem), false $z$-axis orientation, or reconstruction of a symmetric shape from an unsymmetrical specimen (Streicher et al. 1997; Malandain et al. 2004).

The originally published setup for episcopic microscopy with on-block staining has proven valuable for the acquisition of high-quality volume data (Weninger et al. 1998). It, however, seemed rather complex: a dissection microscope with a ring-light was placed perpendicular to the cut surface of the block, and a video-camera connected to a computer was operated by a custom software macro for image acquisition. Later efforts pushed episcopic microscopy to higher resolution (high-resolution episcopic microscopy, HREM, and related episcopic imaging methods; Weninger et al. 2006; Mohun and Weninger 2012a, b; Geyer et al. 2014). HREM is a fluorescence based, automated approach to episcopic microscopy that requires a specialized setup and plastic resin embedding; both limiting its application to certain laboratory settings and object sizes. Automation in sectioning and image capturing is certainly highly desirable for high-throughput applications but also requires higher investment in specialized technical equipment not needed in many other applications.

Our approach herein aims to use standard equipment commonly present in histology laboratories, to assemble a setup that is simple, straightforward, and versatile without sacrificing resolution. In the following, we propose a setup for episcopic microtomy with block surface staining that harnesses the high resolution of contemporary DSLR cameras. We test the performance of the setup and describe a workflow of image acquisition and processing to create high-quality surface models. Our approach neither strives for full automation nor for perfectly aligned image stacks; rather, time investment in manual operation of the microtome and image acquisition, and digital alignment replaced more complex hardware solutions. We applied fiducial markers to counter alignment artifacts and statistically assessed the alignment quality. 


\section{Materials and methods}

\section{Specimen and embedding}

A museum specimen of Alytes obstetricans (Laurenti, 1768) (Amphibia: Anura;

ZMH A12442; snout-vent-length $28 \mathrm{~mm}$ ) was chosen as test specimen. The head, arms, and trunk of the specimen were partly skinned to allow for a better light transmission deep into the sample. The further steps of paraffin (Paraplast Plus ${ }^{\circledR}$; Leica Biosystems) embedding were adopted from Weninger et al. (1998). Most importantly the specimen was impregnated with lead ions prior to paraffin infiltration. The lead ions accumulate in the tissues and allow for later in situ staining of the cut surface of the block (Hegre and Brashear 1946, 1947). The protocol is given in Online Ressource 1.

\section{Setup for episcopic microtomy}

The paraffin block was mounted (melted) onto a wooden base block and clamped into a manually driven rotatory microtome (Microm ${ }^{\mathrm{TM}} \mathrm{HM} 340 \mathrm{E}$; Microm International GmbH). The block was oriented to ensure transverse sectioning of the specimen. The fastened block was moved to a position close to the upper turning point of the microtome. This zero position was marked on the microtome hand wheel for subsequent image acquisition. We used a position slightly before peak block position in order to have space for adjustments of the area captured by the camera in case the area of interest shifted relative to the camera view during sectioning.

A 24 megapixel digital APS-C sensor camera (Nikon D7200 ${ }^{\circledR}$; Nikon Corporation) equipped with a macro lens (Nikon AF-S VR Micro-Nikkor ${ }^{\circledR} 105$ mm 1:2,8G IF-ED) was mounted on a focusing rack (Manfrotto $\left.{ }^{\circledR} 454\right)$, which in turn was fastened to a tripod (Gitzo ${ }^{\circledR}$ G2220). The optical axis of the camera was leveled perpendicular to the block surface. The camera alignment was done by eye, as this proved to be precise enough in previous episcopic imaging trials (unpubl. data). A UV filter and lens hood were mounted to protect the lens from chemicals and paraffin shavings, to enhance contrast, and to prevent lens flare.

One Nikon SB-R200 Wireless Speedlight was placed on each side of the microtome at level with the specimen block in its zero position. Flashes were triggered in TTL mode by the built-in flash of the camera as commander. Flash position was slightly behind the cut surface to mostly illuminate deep into the block. Careful placement of the flashes was essential to reduce glare and reflections from the cut block surface and to lighten up dark areas behind the cut surface plane. This backlit effect enhanced the clarity of the region of interest in the block surface plane. The setup is shown in figure 2. 
We operated the camera in manual focus mode with constant magnification in order to achieve identical pixel sizes (i.e., real world pixel equivalents) for all images. The lens magnification was adjusted such that the field of view covered an area big enough for the expected maximum cross section area of the specimen to fit in, without adding too much empty space around it. The aim was to maximize areal coverage of the specimen projection on the camera sensor and, thus, maximize specimen spatial resolution. Metric-grid paper placed in the focus plane helped to estimate the proper field of view adjustment.

\section{Block sectioning and imaging}

Focusing on the cut block surface was done manually with the focusing rack (lens settings/magnification kept constant). A self-timer and a mirror pre-release were set for the camera to reduce vibrations during image capture. Once all camera settings were set, an image of grid-paper placed in the focus plane was taken (uncompressed RAW format [NEF]) to allow for pixel size calibration.

Fiducial markers were applied to the cut block surface: multiple holes were punched into the block with a pin to create markers for later image alignment (also see, e.g., Malandain et al. 2004 and references therein). Holes were placed in each corner of the field of view using a wood block with a tight, perpendicular drilled hole as guide for the inserted pin. To counter slight deviations from perpendicularity the holes in the upper right and lower left corner were applied with the same guide block orientation whereas those in the other corners were given the opposite orientation (woodblock turned $180^{\circ}$ around the long axis of the pin). The holes were filled with melted dark chocolate (non-toxic, excellent cutting properties) to gain high contrast to the paraffin block. This resulted in subcircular chocolate spots around the specimen on the images. At the beginning, four holes were made into the paraffin, one in each corner. The holes were up to $18 \mathrm{~mm}$ deep and new holes were added as needed. As it was not possible to fill the entire length of holes with chocolate, but just about $1 \mathrm{~mm}$ at once, chocolate had to be added during sectioning. Additional holes were applied for redundancy, in case the chocolate content in another hole ended before it was refilled; in total up to six holes per corner were created.

During block sectioning, images of each pristine cut surface were taken first. Then, a solution of sodium sulfide $\left(\mathrm{Na}_{2} \mathrm{~S} ; 7 \%\right)$ was applied to the cut surface for about $20 \mathrm{~s}$ using a sponge applicator. The sodium sulfide reacted with the lead ions accumulated in tissues and stained tissues of the specimen dark against the surrounding unchanged paraffin (Hegre and Brashear 1946, 1947). The degree of staining was controlled visually. When the surface was considered to be dark enough, superfluous solution was removed with another sponge 
applicator. Then the stained cut surface was photographed in unchanged $x$ - $y$ position, so that the images of the unstained and stained condition were perfectly congruent. The break of the microtome could have been used to fix the block in its position during staining and image acquisition, but this was not necessary as the sponge applicator imposed only minor forces on the cut surface so that the block did not move between unstained and stained images. After taking this pair of images, three sections (10 $\mu \mathrm{m}$ thickness each) of the block surface were cut off. The block was then carefully set to zero position and the next image pair was captured. Care was taken to keep pictures of unstained and stained block surface always alternating in the sequence of images, because this facilitate subsequent automated sorting of images into separate stacks. The specimen was only sectioned in the region of interest, here the anterior part of the body. In this particular project we saved sections on glass slides and stained them with Azan stain (Mulisch and Welsch 2010, with modifications) for future reference. In the pectoral girdle region, the section before the sodium-sulfide-stained and imaged section was saved; anterior to the pectoral girdle, sections were saved at lager intervals as this region was not of particular interest. If it was not possible to save the intended section, e.g., because the section got damaged during mounting, the section before or after (a sodium sulfide stained one) it was saved. Two stained histological sections were digitized for comparison with episcopic images (Leica DM6000 B; Leica Microsystems GmbH). The images were processed (cropping, background cleaning, adjusting of brightness and contrast) in Fiji (based on ImageJ 1.51j; Schindelin et al. 2012; Schneider et al. 2012) and arranged in Adobe ${ }^{\circledR}$ Illustrator ${ }^{\circledR}$ CS6 (version 16.0.3; Adobe ${ }^{\circledR}$ Systems Software).

\section{Digital image processing and pre-alignment}

Image RAW files were converted to TIFF in IrfanView ${ }^{\circledR}$ (version 4.41; Irfan Skiljan, http://www.irfanview.com). The images of unstained and stained block surfaces were opened as separate stacks in Fiji. The size of the pixels was calculated based on the image of metric grid-paper. The differences of corresponding images (stained/unstained pairs) from the two stacks were calculated with Fiji's Image Calculator and the resulting images were inverted. This inverted stack shall be called 'subtracted stack' from here on. Brightness and contrast of the image stacks were adjusted in Fiji.

The stack of stained images was opened in Amira ${ }^{\circledR}$ (version 6.0.1; Konrad-ZuseZentrum Berlin, FEI Visualization Sciences Group) and aligned using the automatic alignment function of the Align Slices module (least squares alignment algorithm). Subsequently, the automatic alignment was checked and corrected manually where needed. The alignment transformations were saved and applied to align the subtracted stack as well. 
By doing so, the subtracted stack was aligned in exactly the same way as the stained stack and corresponding images in both stacks were kept in identical $x-y$ positions. This first alignment was applied to allow the use of the Magic Wand tool with the All slices option for the segmentation of the fiducial spots in later processing steps. These 'pre-aligned' stained and subtracted stacks were then converted to 8-bit gray-scale in Fiji to be compatible with the Segmentation Editor of Amira ${ }^{\circledR}$.

\section{Segmentation, alignment, and assessment of alignment quality}

We selected one fiducial marker in each corner of the aligned stained gray-scale stack (markers I-IV). These selected four markers comprised the spots that later served to test the alignment quality. For each corner, the one fiducial marker was chosen that had the best quality spots (most circular and sharply bordered) and a maximum number of spots through the stack. The spots from the chosen markers were segmented from the aligned gray-scale stained stack as separate materials in Amira ${ }^{\circledR}$ (Magic Wand tool with constantly adjusted thresholds and Brush tool). Marker spots that had no sharp border or were incomplete were omitted during segmentation. This resulted in 164-371 segmented spots per marker canal. The segmentations were stored in a LabelField (subsequently called 'test-spot LabelField') in Amira ${ }^{\circledR}$. The marker's respective spot groups will be referred to as spot groups i-iv (fig. 3 ).

The marker spots of the remaining markers and the specimen were segmented as separate materials (Magic Wand tool with All slices option and selection growing) into a new LabelField. Using this LabelField and the pre-aligned gray-scale stained stack, we created five image stacks with different compositions of elements in their original gray values (Arithmetic module; fig. 3): (1) the specimen only, (2) the specimen and all marker spots except those resulting from markers I-IV, (3) all marker spots except those resulting from markers I-IV, (4) all marker spots of the upper left and lower right corner except those resulting from markers I-IV (marker spots from makers I-IV were included where needed to bridge those images, which lacked other appropriate spots), and (5) one marker spot per corner (two spots in one corner, when we had to switch between markers) not resulting from markers I-IV (marker spots from makers I-IV were included where needed to bridge those images, which lacked other appropriate spots).

These five stacks containing different structure compositions were each aligned in Amira ${ }^{\circledR}$ (alignments 1-5; automatic least squares alignment algorithm; manually corrected if needed). The transformations of each alignment were then applied to a copy of the test-spot LabelField that contained the segmentations of spot groups i-iv. The resulting five differently aligned test-spot LabelFields were exported as tif-image stacks and the midpoint coordinates 
$(x, y$, and $z)$ of each spot in groups $\mathrm{i}-\mathrm{iv}$ were determined using a custom Fiji script resulting in 20 clouds of midpoints (5 alignments with 4 spot groups each).

We used the first principal component of each cloud of midpoints as line of best fit to approximate the long axis of the respective fiducial markers. For each observed point of a given midpoint cloud, the shortest distance of it to the fitted line was calculated. The resulting sets of distances will be called 1.i, 1.ii, through 5.iv depending on the alignment and spot group they belong to. The distance sets were tested for normality (Shapiro-Wilk test). This test did not support the assumption of normality for all sets but two (2.v, 3.iii). A KruskalWallis rank sum tests was performed to test for significant differences between the distance sets. A Dunn's tests with a $p$-value adjustment according to Holm was run to reveal pairwise differences between distance sets, because the Kruskal-Wallis test indicated significant differences. Line fittings, distance calculations, and statistical tests were performed in $\mathrm{R}$ (version 3.3.2; R Core Team 2016) using various functions of the stats package, and the Dunn's test implemented in the PMCMR package (Pohlert 2014) via RStudio ${ }^{\circledR}$ (version 1.0.136; RStudio Team 2016). A $p$-value smaller than 0.05 was considered significant for all tests.

The transformations from the alignment that resulted in the statistically best result (alignment 5) were then used to re-align the pre-aligned gray-scale stacks of the stained and subtracted stacks in Amira ${ }^{\circledR}$. The steps of data alignment and statistical analyses are illustrated in figure 4. Skeletal elements of the right pectoral girdle were segmented (Brush tool with interpolation of up to 5 images) in the re-aligned, final gray-scale stacks. We used both, the stained and the subtracted stack, for segmentation, switching back and forth between them. A scale (five millimeter of the grid-paper image) and selected additional skeletal elements were segmented without distinguishing between bone and cartilage.

\section{Surface generation and processing}

The segmented structures were exported out of Amira ${ }^{\circledR}$ as separate polygon surfaces (objfiles) using a custom Amira ${ }^{\circledR}$ macro (see MultiExport macro and macro documentation in Online Resource 2): all voxel belonging to a given structure/material were extracted (Arithmetic module) and a Isosurface was created. Subsequently, a polygon surface was computed (ExtractSurface module). A copy of the surface was exported (subsequently called 'original surface') and it was reduced in polygon count (SimplificationEditor) and smoothed (SmoothSurface module). Polygon count reduction and smoothing were done in small iterative steps to prevent artifacts in the surface. Finally, the simplified surface was exported. 
Subsequent steps of surface processing were adopted from established workflows (e.g., Kleinteich et al. 2008; Friedrich et al. 2015; Mekonen et al. 2015; Gan et al. 2016). The simplified surfaces were imported into MeshLab (version 1.3.3; Visual Computing Lab-ISTICNR; http://meshlab.sourceforge.net/) and further reduced in polygon count (Quadric Edge Collapse Decimision), smoothed (Taubin Smooth), and cleaned (Compact faces, Compact vertices, Merge Close Vertices, Remove Duplicate Faces, Remove Duplicated Vertex). Surfaces were then passed to MODO ${ }^{\circledR}$ (version 10.1v2; The Foundry; see, e.g., Ablan 2008). The shape of each simplified surface was manually corrected (Move, Smooth, Surface Pen) using the corresponding original surface as template, as smoothing and polygon count reduction altered the shape of the simplified surface and caused artifacts such as holes. The original surface of the scale bar was replaced by an accordingly scaled cube. The cube was positioned in a way that it had the same distance to the camera as the center of the 3D model and that one face was orthogonal to the virtual camera for rendering visualizations. The final surfaces were assigned materials (colors) and the scene was illuminated with Directional and Dome Lights. The rendered images were arranged and labeled in Adobe ${ }^{\circledR}$ Illustrator ${ }^{\circledR}$ CS6 including the replacement of the scale cube by a bar of equivalent length.

\section{Pixel sizes}

We tested different settings of the macro lens and distances of the camera's sensor plane to metric grid-paper to calculate pixel sizes and field of views captured by the camera. We further tested the effect of using a teleconverter (AF-S, TC-14E III; Nikon) and an extension tube (36 mm; Kenko Tokina Co., Ltd.) with the lens because these pieces of equipment are commonly used by photographers and readily available. The pixel size was determined eight times for each distance and lens configuration and the mean pixel size was calculated. Measurements and calculations were done by a custom Fiji macro that required the user to mark a known distance on each grid-paper image eight times.

\section{Results}

\section{Episcopic image stacks and alignment quality}

The partial sectioning of the Alytes specimen resulted in stacks of 620 episcopic images plus one scale image (grid-paper). Image acquisition took about 45 hours including the time for mounting the sections on glass slides. The images had a pixel size of $5.67 \mu \mathrm{m}$ resulting in a voxel size of $5.67 \mu \mathrm{m}$ in $x$ and $y$ and $30 \mu \mathrm{m}$ in $z$. There was some variation in staining intensity among images and within the same image. This resulted in banding when the images 
were rendered as a volume (fig. 3), but had no negative effect on the segmentation or the surfaces creation process (fig. 5). Structures in dark areas of the stained stack (central body parts of the specimen) often showed better contrast in the subtracted stack (fig. 1). The obtained image quality allowed us to well distinguish cartilage and bone tissues, except for the suprascapula cartilage that is partly covered by the very thin cleithrum bone (fig. 1d); the edges of the cleithrum were not traceable with certainty, however it was still possible to roughly segment both structures by comparing subsequent images and approximating borders (fig. 5a,b). In addition to the skeletal structures, muscle tissue, tendons, major nerves, and other organs were clearly distinguishable.

Using different compositions of structures for the stack alignment resulted in different alignment qualities, with statistically significant differences between most distance sets. There, however, was no significant difference between corresponding distance sets of both alignments in which the specimen was part of the structures used for the alignment $(1 \& 2)$. There further were no significant differences between distance sets 3.i \& 5.i, 3.ii \& 5.ii, 3.iii \& 4.iii, 3.iv \& 4.iv, 3.iv \& 5.iv, and 4.iv \& 5.iv, respectively, and some other pairs. Boxand-whisker plots of the different distance sets are given in figure 6; descriptive statistics of the distance sets and the results of Kruskal-Wallis and Dunn's tests are provided in Online Resources 3 and 4, respectively.

\section{Histological serial sections}

The digitized histological sections had a pixel size of $1.67 \mu \mathrm{m}$; smaller pixel sizes would have been achievable with higher magnifications at the Leica slide scanner. The lead impregnation had no visible effect on the tissue integrity and the quality of the Azan staining (fig. 7a). In cases where a sodium sulfide stained section was saved the colors of Azan staining appeared slightly more brownish than those of non-sodium sulfide stained sections (not quantified; fig 7b,d). The histological sections showed all anatomical details usually visible in Azan stained sections. For some of the sections geometric distortions were obvious or some parts of the specimen got damaged or lost (fig. 7a,b).

\section{Pixel sizes}

The smallest calculated pixel size $(2.53 \mu \mathrm{m})$ was obtained using the macro lens with the extension tube. The associated area captured by the camera was $15.19 \mathrm{~mm}$ by $10.13 \mathrm{~mm}$. Other setup variations with their respective pixel sizes and fields of view are given in table 1. 


\section{Discussion}

\section{Limitations, improvements, and strengths}

Our intention was to devise a lab setup for episcopic microscopy with photographic and histological equipment readily accessible in many labs. For our test case, a medium sized frog, the setup worked very well. However, size of the object matters. The minimum and maximum size of a specimen intended for episcopic microtomy is limited by the specifications of the equipment used for sectioning and digital image capture. In our protocol, the specimen is embedded in paraffin and the block has to fit the microtome used. Previously, we sectioned a much bigger toad with a similar protocol, however, on a sliding microtome and the camera above the microtome (unpubl. data). For very large objects sectioning or milling can be a demanding task (e.g., Visible Human Project ${ }^{\circledR}$; Spitzer and Whitlock 1998). Also, the increasing field of view in large objects scales inversely with the spatial resolution. Camera sensor resolution, lens magnification, and lens resolution are to be considered for small specimens. Small objects can be accommodated by inserting extension tubes, teleconverters, or macro bellows (not tested herein) between camera body and lens to increase the magnification; another option is a lens with already high magnification (e.g., Canon MP-E $65 \mathrm{~mm} \mathrm{f} / 2.8$ 1-5x Macro Photo). However, at some point the crystalline fine structure of the paraffin will impose a limit at the lower end of the range; according to our experience we recommend different protocols (resin embedding) for vertebrate objects that are substantially smaller in diameter and for which small pixel sizes are needed $(0.25-0.5 \mu \mathrm{m} \times 0.25-0.5 \mu \mathrm{m}$; Weninger et al. 2006).

Increasing the sensor's pixel density (decreasing the sensor's pixel pitch, i.e., the distance between sensor photo sites) increases the potential camera resolution given the lens has enough resolving power. Note that APS-C DSLR sensors commonly have a higher pixel density than most full frame (FX) sensors and, therefore, an APS-C sensor camera was the better choice for our setup.

Our microtome did not allow the arresting of the block in precisely the same position for consecutive images. Therefore, we obtained stacks of unaligned images. Perfectly aligned stacks can be achieved with other microtomes. We, however, purposely wanted to test our setup and post-sectioning protocols with a microtome type that is more likely to be encountered in many laboratories. Furthermore, we continued using a tripod (easily accessible), although care must be taken that the tripod does not move relative to the specimen block during sectioning. The camera could have been coupled mechanically to the 
microtome by some device, but we did not want to give up simplicity. Rather, we demonstrated that episcopic imaging can be well done with a tripod alone.

Block surface staining with sodium sulfide does not produce consistent staining intensities (Geyer et al. 2009), which, in turn, lead to an inhomogeneous volume (fig. 3). With regard to structure segmentation, this turned out to be advantageous, because some structures were better discernible in lightly stained and others in strongly stained images.

Despite the limitations of the setup, we obtained image stacks that, in our opinion, are of a high quality and very well suitable for morphological work on specimens of this size class. The advantages of episcopically captured image stacks are obvious (Weninger et al. 1998; Geyer et al. 2009): The digitalized cross sections are undistorted, no sections are lost due to processing problems, and the corresponding images in the stained and unstained stacks are congruent allowing for image subtraction and use of both stacks for segmentation in 3D processing software.

\section{Alignment quality}

Our setup requires the virtual alignment of stacked images. Fiducial markers have been shown to be valuable for image stack registration and to overcome known alignment problems such as the "banana" problem (e.g., Toga and Arnicar 1985; Brändle 1989; Ford-Holevinski et al. 1991; Humm et al. 1995; Goldszal et al. 1996; Streicher et al. 1997; Rau et al. 2013). Our results confirmed that the use of external markers can increase alignment quality.

We found no significant difference in the alignment quality of the two alignments (alignments 1-2) that used, among others, the specimen itself for the alignment. When comparing these two alignments, the alignment including the marker spots (2) generally results in better, although not significantly better values (i.e., the distances of the marker spot midpoints to the fitted lines were on average smaller). This clearly demonstrated the influence of the marker spots on the alignment quality. The influence, however, was small possibly because the image area occupied by the marker spots was small compared to the area of the specimen, and both together served for alignment in alignment 2. In other words, the specimen had a higher weight during the alignment process. All alignments that exclusively used various subsets of the marker spots (alignments 3-5) performed statistically better than the two including the specimen (1-2). This indicates that the marker spots provided important alignment information that helped to reduce alignment artifacts.

The alignment using one fiducial marker spot per corner (5) resulted in the statistically best alignment quality; the alignment using all marker spots (3) resulted in second best. The difference between the qualities of these two alignments is based on the significant difference 
of distance sets 3.iii and 5.iii only. This indicates that both these alignments do not differ much in their quality.

In alignment 5, the mean alignment error (in terms of mean distance of midpoints to respectively fitted lines) of spot groups iii-iv was smaller than 3 pixel; this equals about $17 \mu \mathrm{m}$ and was smaller than the image stack resolution in $z$. The highest mean alignment error of this alignment was in spot group ii and equaled $44.7 \mu \mathrm{m}$, approximately 1.5 times the resolution in $z$. The data indicate that there is a slight deviation between the natural shape of the specimen and its' shape after automated alignment. Whether this alignment error is relevant or not, depends on the kind of subsequent analysis and needs to be determined. Episcopic microtomy datasets of our setup may not be suitable for analyses that require high precision measurements such as (geometric) morphometrics.

We observed significant differences between most distance sets within each alignment. This indicates that different parts of the images are aligned with different accuracies. Possible explanations for this observation could be: the marker spots considered for the alignment had different areas and thereby different weights during the alignment process; the holes driven into the block deviated non-uniformly from perpendicularity and led to a shift of the $z$-axis.

We did not analyze the accuracy with which the coordinates of the midpoints were determined. Some errors could have been introduced during the segmentation of the spots chosen to test the alignment quality and during the determination of their midpoint coordinates. Yet, we expect the possible error in midpoint determination to have only minor effect on the alignment comparisons as we used the same test-spot LabelField for all distance calculations.

\section{Alternative steps and software}

There is a large, if not bewildering, number of software packages that can be freely combined to achieve the results desired. Herein we present the solution that suited us best, but alternative packages and workflows do exist. Amira ${ }^{\circledR}$ offers various functions of image processing that we performed in Fiji instead. For surface generation, there is an alternative Amira ${ }^{\circledR}$ module (Generate Surface); a custom script for exporting multiple separate surfaces created with Generate Surface is available from the authors. The Fiji extension TrakEM2 (Cardone et al. 2012) can perform alignment and segmentation tasks. VGStudio MAX ${ }^{\circledR}$ (Volume Graphics GmbH) also offers some functions similar to Amira ${ }^{\circledR}$. Separate image stacks could have been exported for each segmented structure (image stack export included in the macro in Online Resource 2). The resulting image stacks could have been used to create surfaces in, for example, Imaris ${ }^{\circledR}$ (Bitplain) or GOM Inspect (GOM GmbH). GOM Inspect is 
also capable of polygon count reduction, smoothing, and cleaning of the surfaces. For final surface processing and image rendering Maya ${ }^{\circledR}$ (Autodesk) or Blender ${ }^{\circledR}$ (Blender Foundation) are alternative options.

\section{Comparison to other methods of generating volume data}

For acquiring volume data, specimens that are suitable for episcopic microtomy commonly will be suitable for other imaging techniques as well. One approach is histological serial sectioning with subsequent mounting on slides, staining, digitizing, stacking, and alignment (e.g., Handschuh et al. 2010). Microscopic slides can be examined at very high magnifications and contrast between tissues of interests can be enhanced with appropriate staining methods; the episcopic microtomy workflow suggested herein has certain limitations in tissue staining and resolution (also compare fig. 7). The major disadvantage of histological sections with regard to digital 3D reconstruction is that the sections are more or less distorted during the sectioning and mounting process. Episcopic microtomy does not have this problem because the block surface, not the cut section, is digitized. It might be interesting to evaluate the applicability of non-rigid alignment algorithms to elastically register a digitized histological section to a corresponding episcopic image, for example, by adapting the method suggested by Saalfeld et al. (2012) (also see Laan et al. 1989).

Micro-computed tomography is a widely used imaging method in biology (Neues and Epple 2008; Mizutani and Suzuki 2012). It allows the non-destructive acquisition of isotropic, high-resolution (sub-micrometer) volume data. $\mu \mathrm{CT}$ data are inherently aligned. For soft tissue visualization (e.g., muscle), contrast staining may be required (e.g., Metscher 2009a, b; Gignac et al. 2016). Using a contrast agent, however, is no longer non-destructive because the agent might change tissue properties and possibly remains in the specimens for some time (Schmidbaur et al. 2015 and references therein). Even with contrast staining, making cartilage discernible and distinguishable from other tissues remains difficult (unpubl. data).

Histological serial sections and episcopic microtomy are suitable methods for soft tissue and cartilage visualization and may be a better choice than $\mu \mathrm{CT}$ scans depending on both the research question and considerations about the integrity of the specimens.

Magnetic resonance imaging has been used as another non-destructive technique to acquire inherently aligned volume data of specimens (e.g., Arnold et al. 2000; Trunbull and Mori 2007; Driehuys et al. 2008; Gabbay-Benziv et al. 2017). MRI data acquisition is typically performed without physically altering or staining the specimen (but see Rohrer et al. 2005). The resolution, however, is still limited and dependent on magnetic field strength. To our knowledge, voxel sizes down to an edge length of $10 \mu \mathrm{m}$ have been achieved (Lee et al. 
2015). High-resolution MRI scanners are much less accessible to most researchers than $\mu \mathrm{CT}$ scanners or even histological equipment.

Micro-computed tomography and MRI are volume data acquisition methods that have several advantages with regard to shape and specimen preservation, and with regard to speed of data acquisition (several hours versus several days to weeks in episcopic microtomy and histological serial sectioning). $\mu \mathrm{CT}$ and MRI, however, currently have limits in tissue contrast (one color channel) and visibility. Thus it depends on the research goal, which method is most efficient with regard to time and the data quality needed.

Some studies combined different imaging methods (e.g., Laan et al. 1989; Pieles et al. 2007; Schulz et al. 2012; Handschuh et al. 2013; Herdina et al. 2015). This adds advantages and potentially neutralizes the disadvantages of each technique. In our labs, we combined $\mu \mathrm{CT}$ scans (for bones) with subsequent episcopic microtomy (for cartilage and soft tissues) of the same specimen; data sets could be registered to each other (unpubl. data).

\section{Conclusion}

Episcopic microtomy workflows can produce huge amounts of high-quality digital images with excellent differential tissue contrast, at a small inter-image distance, and without the need for physical storage space for glass slides. Selected sections of a specimen can still be preserved on glass slides during the episcopic process if necessary. After applying standard staining (e.g., Azan), these histological sections provide additional anatomical information. Our setup is clearly not suitable for HREM. If resolutions at the cellular level are needed or objects are much smaller than ours, stacking of histological sections (Handschuh et al. 2013) or HREM (Wenigner et al. 2006) need to be considered. Furthermore, our setup is not recommended for projects that need high automation or high throughput. The described setup and procedures, however, can easily be set up by the occasional user to take advantage of episcopic imaging. Our simple episcopic microtomy setup allowed relatively fast specimen processing and the resulting datasets offered ample resolution. Episcopic microtomy has a lot of potential in morphological work pipelines to generate volume data.

\section{Compliance with ethical standards}

\section{Conflict of interest}

The authors declare that they have no conflict of interest. 


\section{Ethical approval}

This article does not contain any studies with human participants or living animals performed by any of the authors. 


\section{References}

Ablan D (2008) The Official Luxology modo Guide. Version 301. Course Technology, Cengage Learning, Boston

Arnold AS, Salinas S, Asakawa DJ, Delp SL (2000) Accuracy of muscle moment arms estimated from MRI-based musculoskeletal models of the lower extremity. Comput Aided Surg 5:108-119. doi: 10.1002/1097-0150(2000)5:2<108::AID-IGS5>3.0.CO;2-2

Born G (1876) Ueber die Nasenhöhlen und den Thränennasengang der Amphibien. Morphol Jahrb 2:577-646

Born G (1883) Die Plattenmodelliermethode. Arch f mikrosk Anat 22:584-599

Brainerd EL, Baier DB, Gatesy SM, Hedrick TL, Metzger KA, Gilbert SL, Crisco JJ (2010) $\mathrm{X}$-ray reconstruction of moving morphology (XROMM): precision, accuracy and applications in comparative biomechanics research. J Exp Zool A Ecol Genet Physiol 313A:262-279. doi: 10.1002/jez.589

Brändle K (1989) A new method for aligning histological serial sections for threedimensional reconstruction. Comput Biomed Res 22:52-62. doi: 10.1016/00104809(89)90015-3

Cardona A, Saalfeld S, Schindelin J, Arganda-Carreras I, Preibisch S, Longair M, Tomancak P, Hartenstein V, Douglas RJ, Samuel A (2012) TrakEM2 software for neural circuit reconstruction. PLoS ONE 7:e38011. doi: 10.1371/journal.pone.0038011

Charles JP, Cappellari O, Spence AJ, Wells DJ, Hutchinson JR (2016) Muscle moment arms and sensitivity analysis of a mouse hindlimb musculoskeletal model. J Anat 229:514-535. doi: $10.1111 /$ joa. 12461

Cox PG, Fagan MJ, Rayfield EJ, Jeffery N (2011) Finite element modelling of squirrel, guinea pig and rat skulls: using geometric morphometrics to assess sensitivity. J Anat 219:696-709. doi: 10.1111/j.1469-7580.2011.01436.x

Curtis N, Jones MEH, Evans SE, Shi J, O'Higgins P, Fagan MJ (2010) Predicting muscle activation patterns from motion and anatomy: modelling the skull of Sphenodon (Diapsida: Rhynchocephalia). J R Soc Interface 7:153-160. doi: 10.1098/rsif.2009.0139

Davies TG, Rahman IA, Lautenschlager S, Cunningham JA, Asher RJ, Barrett PM, Bates KT, Bengtson S, Benson RBJ, Boyer DM, Braga J, Bright JA, Claessens LPAM, Cox PG, 
Dong X, Evans AR, Falkingham PL, Friedman M, Garwood RJ, Goswami A, Hutchinson JR, Jeffery NS, Johanson Z, Lebrun R, Martínez-Pérez C, Marugán-Lobón J, O'Higgins PM, Metscher B, Orliac M, Rowe TB, Rücklin M, Sánchez-Villagra MR, Shubin NH, Smith SY, Starck JM, Stringer C, Summers AP, Sutton MD, Walsh SA, Weisbecker V, Witmer LM, Wroe S, Yin Z, Rayfield EJ, Donoghue PCJ (2017) Open data and digital morphology. Proc. R. Soc. B 284:20170194. doi: 10.1098/rspb.2017.0194

Driehuys B, Nouls J, Badea A, Bucholz E, Ghaghada K, Petiet A, Hedlund LW (2008) Small animal imaging with magnetic resonance microscopy. ILAR J 49:35-53. doi: 10.1093/ilar.49.1.35

Ford-Holevinski TS, Castle MR, Herman JP, Watson SJ (1991) Microcomputer-based threedimensional reconstruction of in situ hybridization autoradiographs. J Chem Neuoanat 4:373-385. doi: 10.1016/0891-0618(91)90044-D

Fortuny J, Marcé-Nogué J, Heiss E, Sanchez M, Gil L, Galobart À (2015) 3D bite modeling and feeding mechanics of the largest living amphibian, the Chinese Giant salamander Andrias davidianus (Amphibia: Urodela). PLoS ONE 10:e0121885. doi: 10.1371/journal.pone.0121885

Friedrich F, Schulz J, Kubiak M, Beckmann F, Wilde F (2015) The larval head anatomy of Rhyacophila (Rhyacophilidae) with discussion on mouthpart homology and the groundplan of Trichoptera. J Morphol 276:1505-1524. doi: 10.1002/jmor.20475

Gabbay-Benziv R, Reece EA, Wang F, Bar-Shir A, Harman C, Turan OM, Yang P, Turan S (2017) A step-wise approach for analysis of the mouse embryonic heart using 17.6Tesla MRI. Magn Reson Imaging 35:46-53. doi: 10.1016/j.mri.2016.08.008

Gan LL, Hertwig ST, Das I, Haas A (2016) The anatomy and structural connectivity of the abdominal sucker in the tadpoles of Huia cavitympanum, with comparisons to Meristogenys jerboa (Lissamphibia: Anura: Ranidae). J Zool Syst Evol Res 54:46-59. doi: 10.1111/jzs.12113

Geyer SH, Mohun TJ, Weninger WJ (2009) Visualizing vertebrate embryos with episcopic 3D imaging techniques. ScientificWorldJOURNAL 9:1423-1437. doi: $10.1100 /$ tsw.2009.154

Geyer SH, Nöhammer MM, Mathä M, Reissig L, Tinhofer IE, Weninger WJ (2014) HighResolution Episcopic Microscopy (HREM): a tool for visualizing skin biopsies. Microsc Microanal 20:1356-1364. doi: 10.1017/S1431927614013063 
Gignac PM, Kley NJ, Clarke JA, Colbert MW, Morhardt AC, Cerio D, Cost IN, Cox PG, Daza JD, Early CM, Echols MS, Henkelman RM, Herdina AN, Holliday CM, Li Z, Mahlow K, Merchant S, Müller J, Orsbon CP, Paluh DJ, Thies ML, Tsai HP, Witmer LM (2016) Diffusible iodine-based contrast-enhanced computed tomography (diceCT): an emerging tool for rapid, high-resolution, 3-D imaging of metazoan soft tissues. J Anat 228:889-909. doi: 10.1111/joa.12449

Golding RE, Ponder WF, Byrne M (2009) Three-dimensional reconstruction of the odontophoral cartilages of Caenogastropoda (Mollusca: Gastropoda) using micro-CT: Morphology and phylogenetic significance. J Morphol 270:558-587. doi: 10.1002/jmor.10699

Goldszal AF, Tretiak OJ, Liu DD, Hand PJ (1996) Multimodality multidimensional image analysis of cortical and subcortical plasticity in the rat brain. Ann Biomed Eng 24:430 439. doi: 10.1007/BF02660892

Griep S, Schilling N, Marshall P, Amling M, Hahne LM, Haas A (2013) Pectoral girdle movements and the role of the glenohumeral joint during landing in the toad, Rhinella marina (Linnaeus, 1758). Zoomorphology 132:325-338. doi: 10.1007/s00435-013-0189-0

Gunz P, Ramsier M, Kuhrig M, Hublin J, Spoor F (2012) The mammalian bony labyrinth reconsidered, introducing a comprehensive geometric morphometric approach. J Anat 220:529-543. doi: 10.1111/j.1469-7580.2012.01493.x

Haas A (2001) Mandibular arch musculature of anuran tadpoles, with comments on homologies of amphibian jaw muscles. J Morphol 247:1-33. doi: 10.1002/10974687(200101)247:1<1::AID-JMOR1000>3.0.CO;2-3

Hammel JU, Jahn H, Möckel L, Herzen J, Greving I, Wilde F, Beckmann F, Nickel M (2013) Computational fluid dynamics of sponge aquiferous systems. HASYLAB Annual Report

Handschuh S, Baeumler N, Schwaha T, Ruthensteiner B (2013) A correlative approach for combining microCT, light and transmission electron microscopy in a single 3D scenario. Front Zool 10:44. doi: 10.1186/1742-9994-10-44

Handschuh S, Schwaha T, Metscher BD (2010) Showing their true colors: a practical approach to volume rendering from serial sections. BMC Dev Biol 10:41. doi: $10.1186 / 1471-213 X-10-41$ 
Hegre ES, Brashear AD (1946) Block-surface staining. Stain Technol 21:161-164. doi: $10.3109 / 10520294609110063$

Hegre ES, Brashear AD (1947) The block-surface method of staining as applied to the study of embryology. Anat Rec 97:21-28. doi: 10.1002/ar.1090970103

Heiss E, Handschuh S, Aerts P, van Wassenbergh S (2016) Musculoskeletal architecture of the prey capture apparatus in salamandrid newts with multiphasic lifestyle: does anatomy change during the seasonal habitat switches? J Anat:757-770. doi: 10.1111/joa.12445

Henne S, Friedrich F, Hammel JU, Sombke A, Schmidt-Rhaesa A (2017) Reconstructing the anterior part of the nervous system of Gordius aquaticus (Nematomorpha, Cycloneuralia) by a multimethodological approach. J Morphol 278:106-118. doi: 10.1002/jmor.20623

Herdina AN, Plenk H, Benda P, Lina PHC, Herzig-Straschil B, Hilgers H, Metscher BD (2015) Correlative 3D-imaging of Pipistrellus penis micromorphology: validating quantitative microCT images with undecalcified serial ground section histomorphology. $\mathrm{J}$ Morphol 276:695-706. doi: 10.1002/jmor.20372

Humm JL, Macklis RM, Lu XQ, Yang Y, Bump K, Beresford B, Chin LM (1995) The spatial accuracy of cellular dose estimates obtained from 3D reconstructed serial tissue autoradiographs. Phys Med Biol 40:163-180

Joschko M, Graff O, Müller PC, Kotzke K, Lindner P, Pretschner DP, Larink O (1991) A non-destructive method for the morphological assessment of earthworm burrow systems in three dimensions by X-ray computed tomography. Biol Fertil Soils 11:88-92. doi: 10.1007/BF00336369

Kargo WJ, Rome LC (2002) Functional morphology of proximal hindlimb muscles in the frog Rana pipiens. J Exp Biol 205:1987-2004

Kleinteich T, Beckmann F, Herzen J, Summers AP, Haas A (2008) Applying x-ray tomography in the field of vertebrate biology: form, function, and evolution of the skull of caecilians (Lissamphibia: Gymnophiona). Proc SPIE 7078:70780D. doi: $10.1117 / 12.795063$

Kleinteich T, Maddin HC, Herzen J, Beckmann F, Summers AP (2012) Is solid always best? Cranial performance in solid and fenestrated caecilian skulls. J Exp Biol 215:833-844. doi: 10.1242/jeb.065979 
Laan AC, Lamers WH, Huijsmans DP, Kortschot AT, Smith J, Strackee J, Los JA (1989)

Deformation-corrected computer-aided three-dimensional reconstruction of immunohistochemically stained organs: application to the rat heart during early organogenesis. Anat Rec 224:443-457. doi: 10.1002/ar.1092240313

Lautenschlager S, Bright JA, Rayfield EJ (2014) Digital dissection - using contrast-enhanced computed tomography scanning to elucidate hard- and soft-tissue anatomy in the Common Buzzard Buteo buteo. J Anat 224:412-431. doi: 10.1111/joa.12153

Lee CH, Blackband SJ, Fernandez-Funez P (2015) Visualization of synaptic domains in the Drosophila brain by magnetic resonance microscopy at 10 micron isotropic resolution. Sci Rep 5:1234168. doi: 10.1038/srep08920

Malandain G, Bardinet É, Nelissen K, Vanduffel W (2004) Fusion of autoradiographs with an MR volume using 2-D and 3-D linear transformations. NeuroImage 23:111-127. doi: 10.1016/j.neuroimage.2004.04.038

McGill R, Tukey JW, Larsen WA (1978) Variations of box plots. Am Stat 32:12. doi: $10.2307 / 2683468$

Mekonen HK, Hikspoors JPJM, Mommen G, Köhler SE, Lamers WH (2015) Development of the ventral body wall in the human embryo. J Anat 227:673-685. doi: 10.1111/joa.12380

Metscher BD (2009a) MicroCT for comparative morphology: simple staining methods allow high-contrast 3D imaging of diverse non-mineralized animal tissues. BMC Physiol 9:114. doi: 10.1186/1472-6793-9-11

Metscher BD (2009b) MicroCT for developmental biology: a versatile tool for high-contrast 3D imaging at histological resolutions. Dev Dyn 238:632-640. doi: 10.1002/dvdy.21857

Mizutani R, Suzuki Y (2012) X-ray microtomography in biology. Micron 43:104-115. doi: 10.1016/j.micron.2011.10.002

Mohun TJ, Weninger WJ (2012a) Episcopic three-dimensional imaging of embryos. Cold Spring Harb Protoc 2012:641-646. doi: 10.1101/pdb.top069567

Mohun TJ, Weninger WJ (2012b) Generation of volume data by episcopic three-dimensional imaging of embryos. Cold Spring Harb Protoc 2012:681-682. doi:

10.1101/pdb.prot069591

Mulisch M, Welsch U (eds) (2010) Romeis Mikroskopische Technik, 18th edn. Spektrum Akademischer Verlag, Heidelberg 
Neues F, Epple M (2008) X-ray microcomputer tomography for the study of biomineralized endo- and exoskeletons of animals. Chem Rev 108:4734-4741. doi: 10.1021/cr078250m

Odgaard A, Andersen K, Melsen F, Gundersen HJ (1990) A direct method for fast threedimensional serial reconstruction. J Microsc 159:335-342. doi: 10.1111/j.13652818.1990.tb03038.x

Pieles G, Geyer SH, Szumska D, Schneider J, Neubauer S, Clarke K, Dorfmeister K, Franklyn A, Brown SD, Bhattacharya S, Weninger WJ (2007) $\mu$ MRI-HREM pipeline for highthroughput, high-resolution phenotyping of murine embryos. J Anat 211:132-137. doi: 10.1111/j.1469-7580.2007.00746.x

Piras P, Sansalone G, Teresi L, Moscato M, Profico A, Eng R, Cox TC, Loy A, Colangelo P, Kotsakis T (2015) Digging adaptation in insectivorous subterranean eutherians. The enigma of Mesoscalops montanensis unveiled by geometric morphometrics and finite element analysis. J Morphol 276:1157-1171. doi: 10.1002/jmor.20405

Pohlert T (2014) The pairwise multiple comparison of mean ranks package (PMCMR). R package. http://CRAN.R-project.org/package=PMCMR

R Core Team (2016) R: A language and environment for statistical computing. Version 3.3.2. R Foundation for Statistical Computing. https://www.R-project.org/

Rahman IA, Darroch SAF, Racicot RA, Laflamme M (2015) Suspension feeding in the enigmatic Ediacaran organism Tribrachidium demonstrates complexity of Neoproterozoic ecosystems. Science Advances 1:e1500800. doi: 10.1126/sciadv.1500800

Rau TS, Würfel W, Lenarz T, Majdani O (2013) Three-dimensional histological specimen preparation for accurate imaging and spatial reconstruction of the middle and inner ear. Int J Comput Assist Radiol Surg 8:481-509. doi: 10.1007/s11548-013-0825-7

Rohrer M, Bauer H, Mintorovitch J, Requardt M, Weinmann H (2005) Comparison of magnetic properties of MRI contrast media solutions at different magnetic field strengths. Invest Radiol 40:715-724. doi: 10.1097/01.rli.0000184756.66360.d3

RStudio Team (2016) RStudio: Integrated Development for R. Version 1.0.136. RStudio, Inc. http://www.rstudio.com/

Saalfeld S, Fetter R, Cardona A, Tomancak P (2012) Elastic volume reconstruction from series of ultra-thin microscopy sections. Nat Methods 9:717-720. doi:

10.1038/nmeth.2072 
Schindelin J, Arganda-Carreras I, Frise E, Kaynig V, Longair M, Pietzsch T, Preibisch S, Rueden C, Saalfeld S, Schmid B, Tinevez J, White DJ, Hartenstein V, Eliceiri K, Tomancak P, Cardona A (2012) Fiji: an open-source platform for biological-image analysis. Nat Methods 9:676-682. doi: 10.1038/nmeth.2019

Schmidbaur H, Keklikoglou K, Faulwetter S, Metscher BD (2015) Exploring methods to remove iodine and phosphotungstic acid stains from zoological specimens. Proc Bruker MicroCT user meeting 2015:116-123

Schneider CA, Rasband WS, Eliceiri KW (2012) NIH image to ImageJ: 25 years of image analysis. Nat Methods 9:671-675. doi: 10.1038/nmeth.2089

Schulz G, Waschkies C, Pfeiffer F, Zanette I, Weitkamp T, David C, Müller B (2012) Multimodal imaging of human cerebellum - merging X-ray phase microtomography, magnetic resonance microscopy and histology. Sci Rep 2:69. doi: 10.1038/srep00826

Spitzer VM, Whitlock DG (1998) The visible human dataset: the anatomical platform for human simulation. Anat Rec 253:49-57. doi: 10.1002/(SICI)10970185(199804)253:2<49::AID-AR8>3.0.CO;2-9

Staedler YM, Masson D, Schönenberger J, Sun M (2013) Plant tissues in 3D via X-ray tomography: simple contrasting methods allow high resolution imaging. PLoS ONE 8:e75295. doi: 10.1371/journal.pone.0075295

Streicher J, Weninger WJ, Müller GB (1997) External marker-based automatic congruencing: a new method of 3D reconstruction from serial sections. Anat Rec 248:583-602

Toga A, Arnicar T (1985) Image analysis of brain physiology. IEEE Comput Graph Appl 5:20-25. doi: 10.1109/MCG.1985.276259

Turnbull DH, Mori S (2007) MRI in mouse developmental biology. NMR Biomed 20:265274. doi: $10.1002 / \mathrm{nbm} .1146$

Weninger WJ, Geyer SH, Mohun TJ, Rasskin-Gutman D, Matsui T, Ribeiro I, Costa LdF, Izpisúa-Belmonte JC, Müller GB (2006) High-resolution episcopic microscopy: a rapid technique for high detailed 3D analysis of gene activity in the context of tissue architecture and morphology. Anat Embryol 211:213-221. doi: 10.1007/s00429-0050073-x 
Weninger WJ, Meng S, Streicher J, Müller GB (1998) A new episcopic method for rapid 3-D reconstruction: applications in anatomy and embryology. Anat Embryol 197:341-348. doi: $10.1007 / \mathrm{s} 004290050144$

Werneburg I, Wilson LAB, Parr WCH, Joyce WG (2015) Evolution of neck vertebral shape and neck retraction at the transition to modern turtles: an integrated geometric morphometric approach. Syst Biol 64:187-204. doi: 10.1093/sysbio/syu072 


\section{Figures}

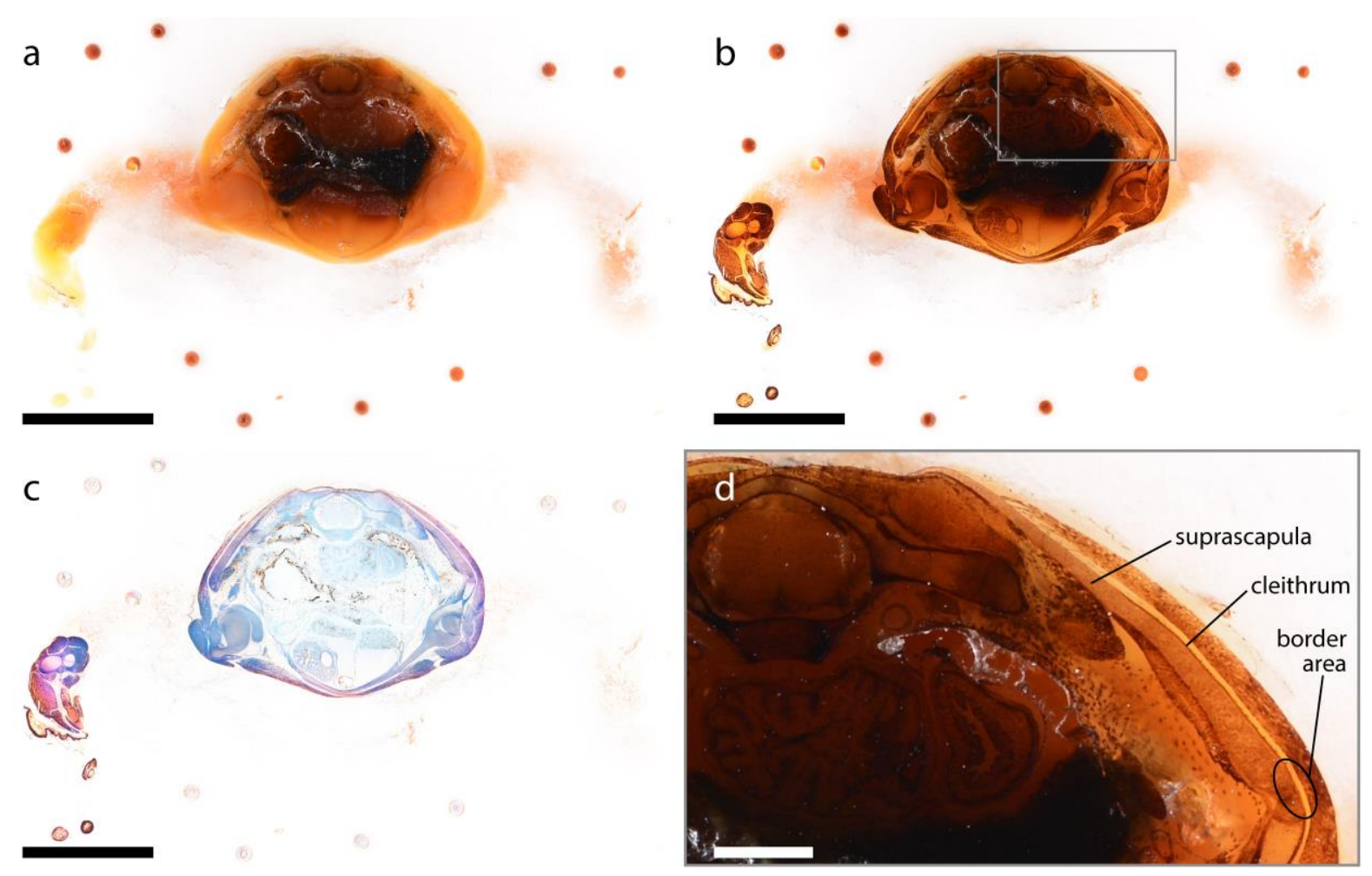

Fig. 1 Cross section through the pectoral girdle of Alytes obstetricans (ZMH A12442).

Episcopic images (cropped) of unstained (a) and stained (b) cut surface of the paraffin

block. c: Result of inverted difference of images a and b. d: Detail of b showing no clear border of cleithrum. Black scales: $5 \mathrm{~mm}$; white scale: $1 \mathrm{~mm}$

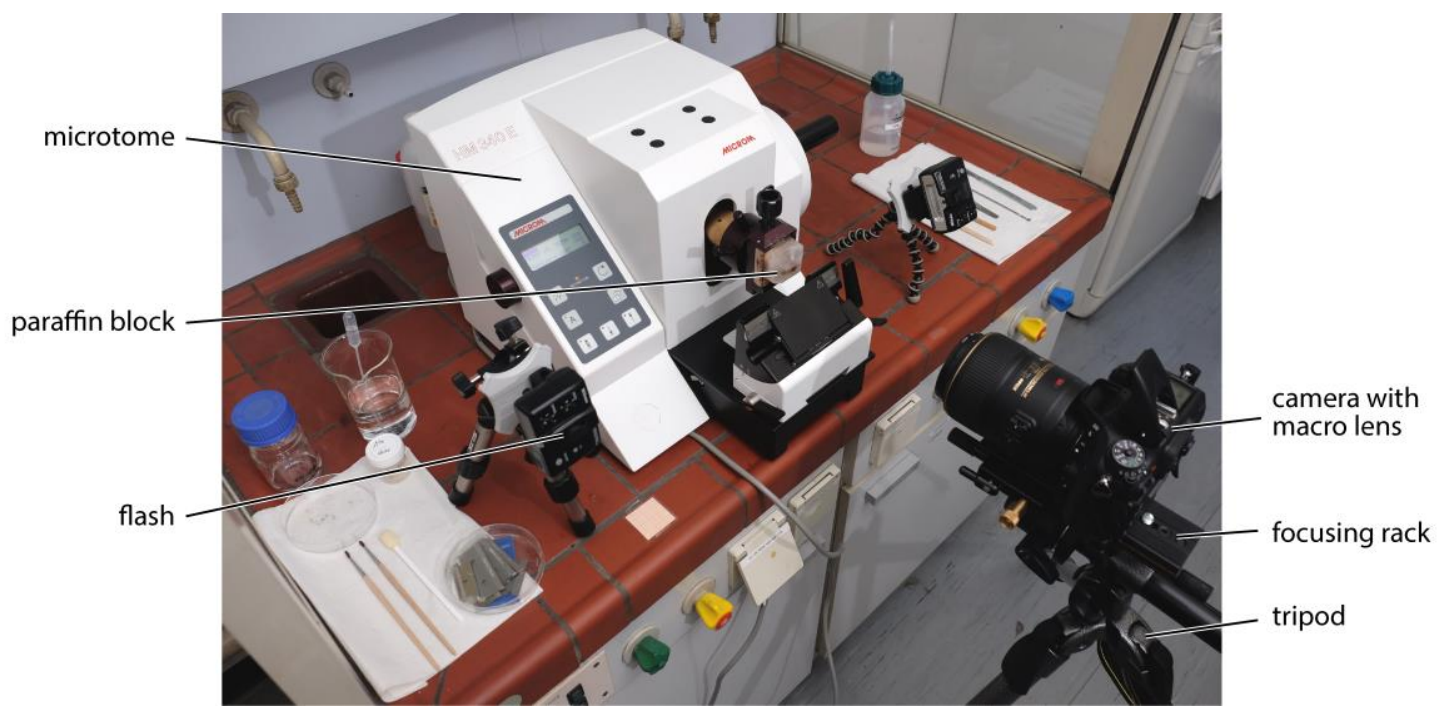

Fig. 2 Setup for episcopic microtomy, lens hood removed 


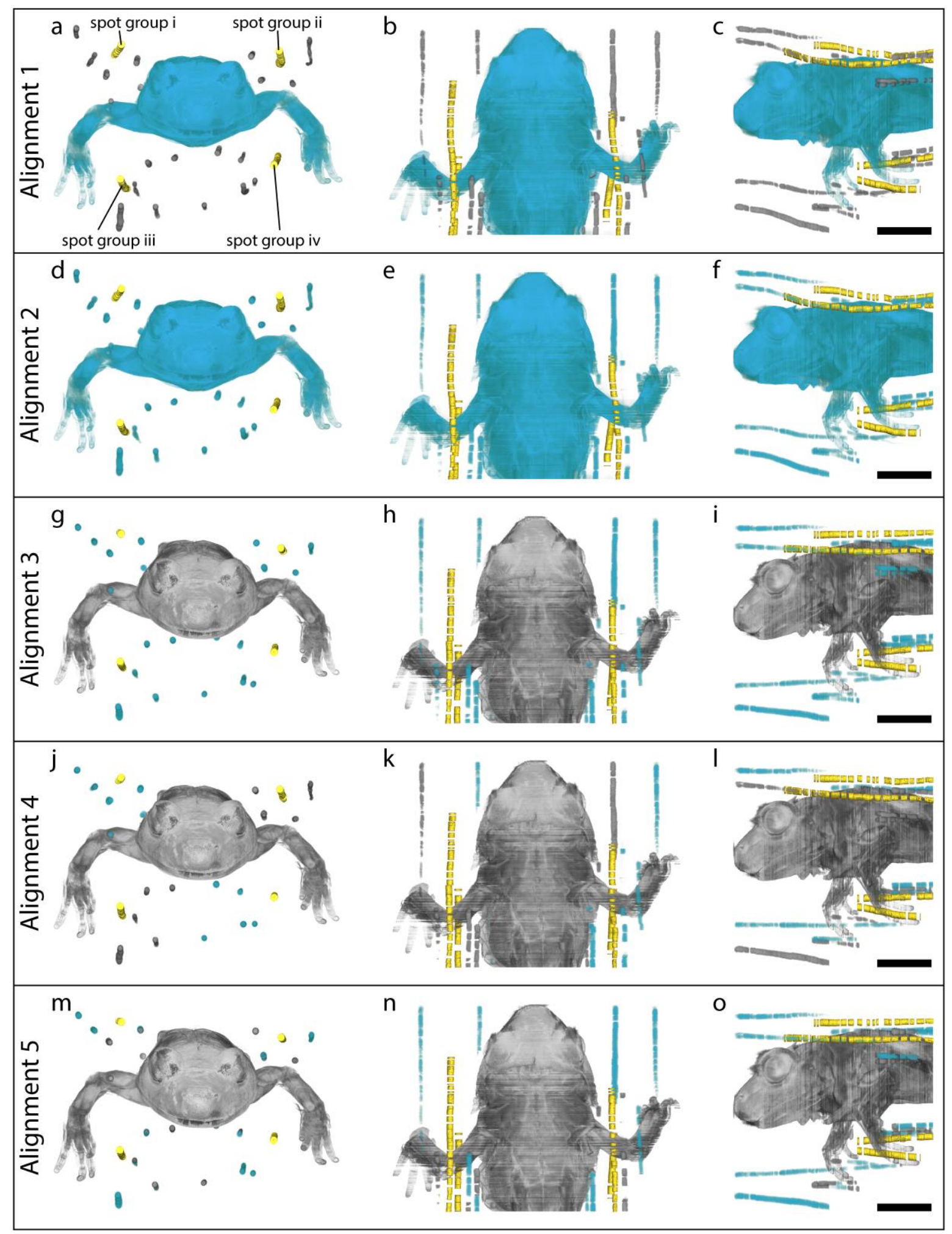

Fig. 3 3D visualizations of differently aligned stained image stacks (converted to gray-scale) with the structures belonging to different alignments highlighted. Views from left to right: anterior, dorsal, lateral. Scale: $5 \mathrm{~mm}$; all views at same scale. Blue: volume rendering of structure used for alignment; yellow: Isosurface of test-spot segmentations aligned accordingly; gray: volume rendering of remaining structures. a-c: Alignment 1. d-f: Alignment 2. g-i: Alignment 3. j-1: Alignment 4. m-o: Alignment 5 


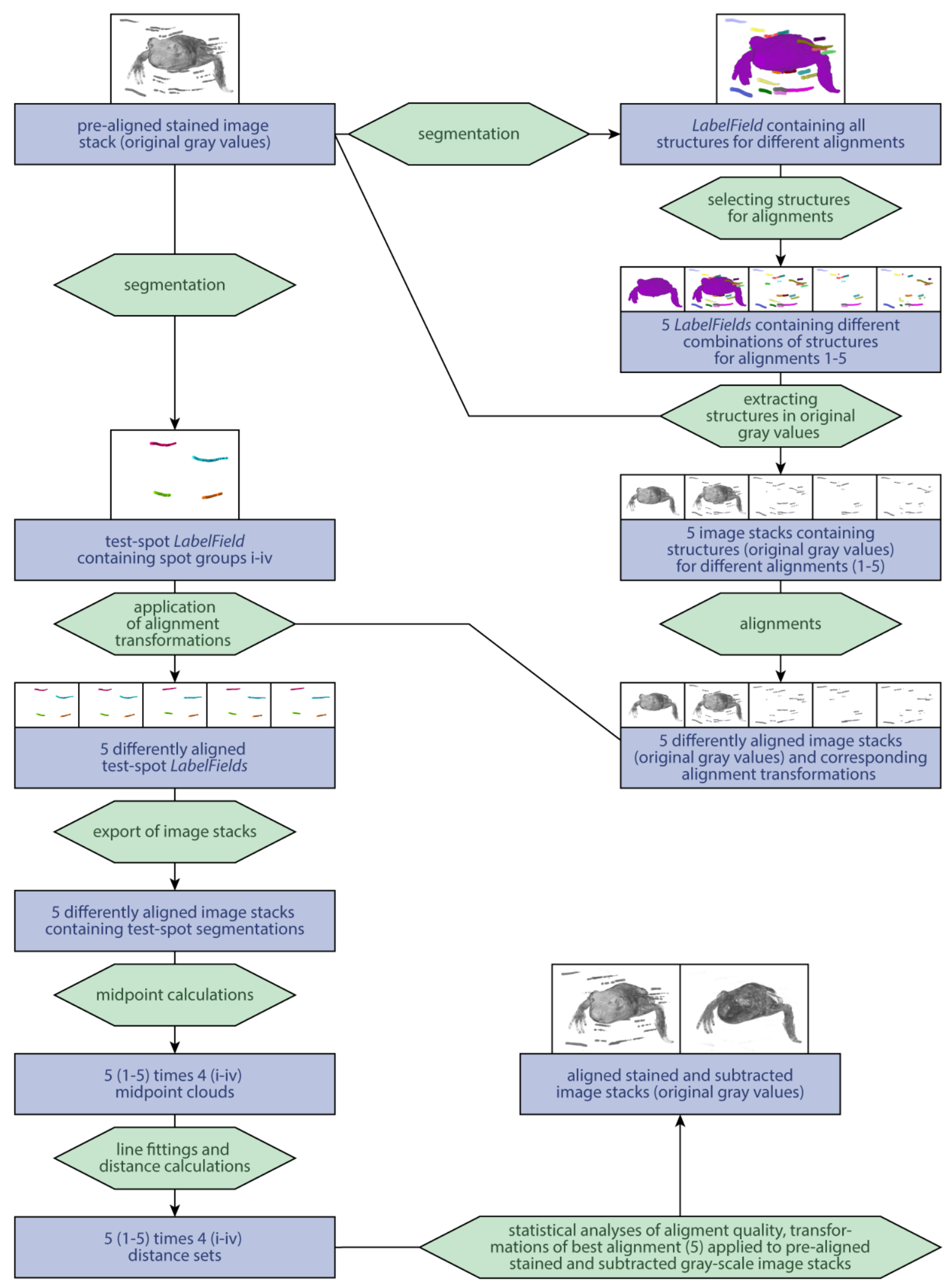

Fig. 4 Steps of data alignment and statistical analysis of alignment quality 


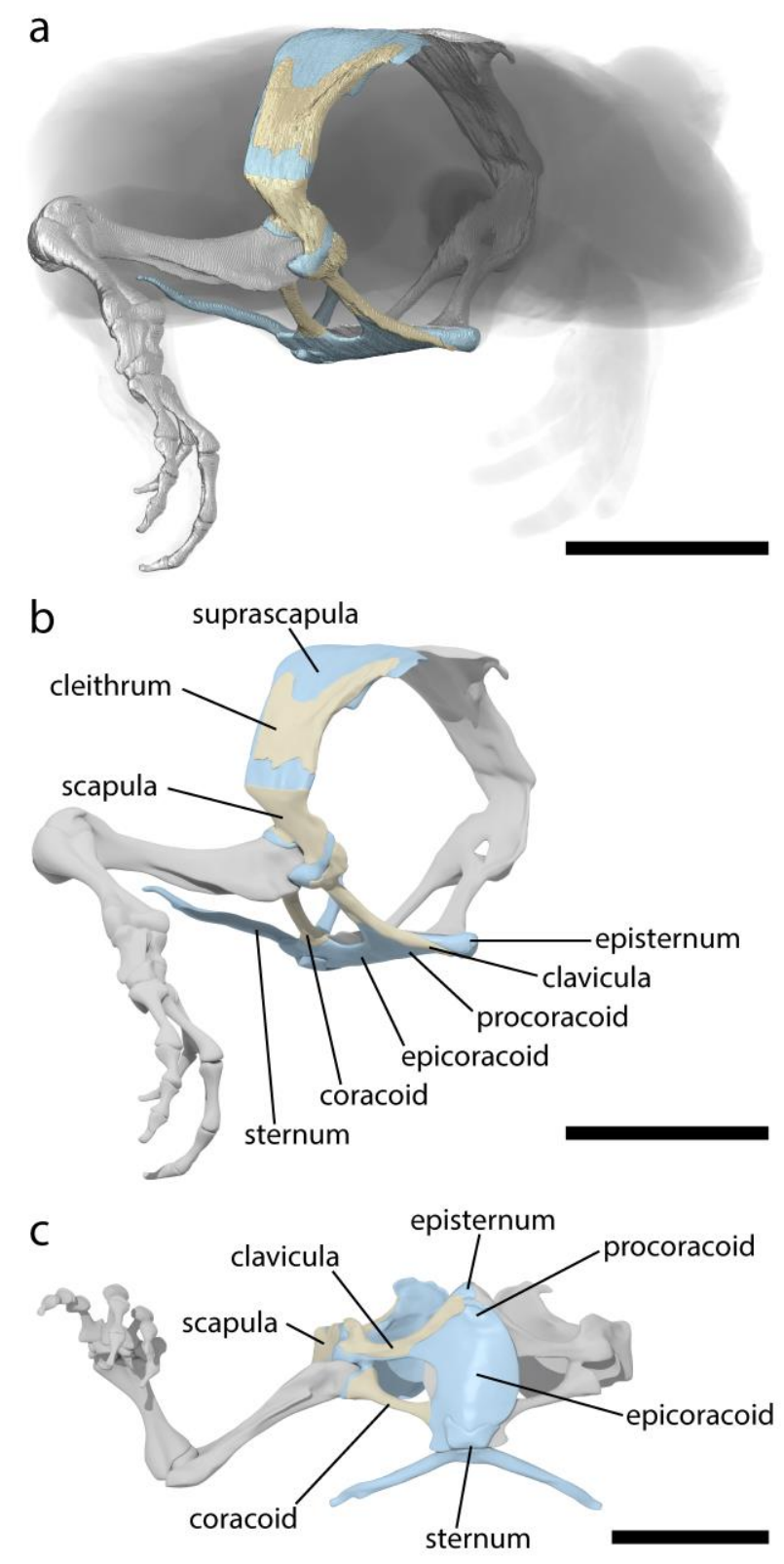

Fig. 5 a: Unprocessed Isosurfaces of the segmented structures (pectoral girdle and additional structures) in a volume rendering (converted to uniform gray-scale) of the Alytes obstetricans specimen (ZMH A12442; image created in Amira ${ }^{\circledR}$ ). The surfaces appear stepped due to an inter-image distance of $30 \mu \mathrm{m}$. Anterolateral view, anterior to the right. Beige: bone; blue: cartilage; gray: additional skeleton without distinguishing bone and cartilage. Scale: $5 \mathrm{~mm}$. b, c: Simplified, smoothed, and corrected surface model of the surfaces in a (image rendered in $\mathrm{MODO}^{\circledR}$ ). Anterolateral (b) and ventral (c) views. Same color code as in a, scales $5 \mathrm{~mm}$ 


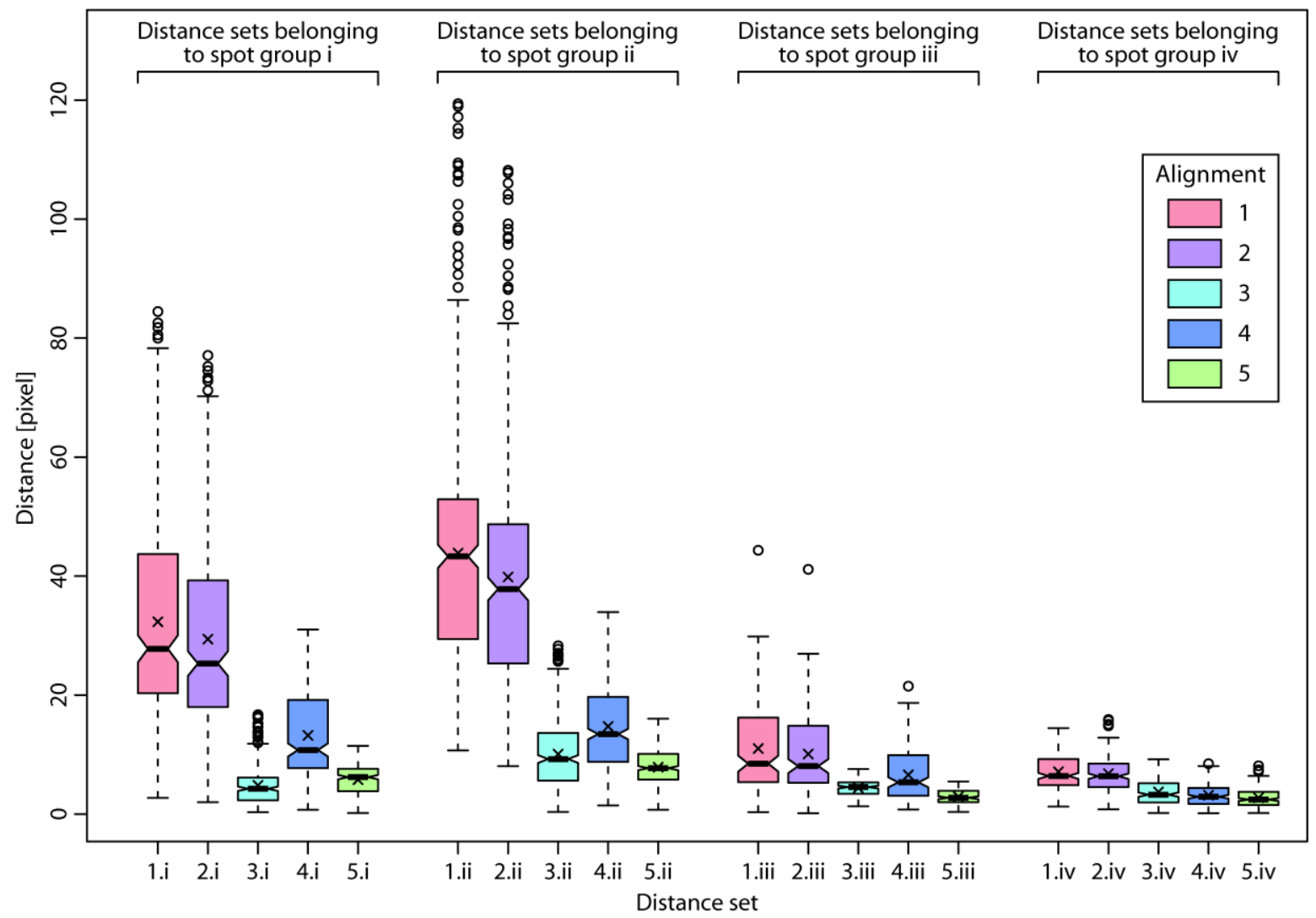

Fig. 6 Notched box-and-whisker plots of distance sets grouped by the respective spot group they belong to. If notches of two boxes do not overlap, this is strong evidence that there is a true difference in the means of the respective distance sets (McGill et al. 1978).

Circle: potential outlier; cross: mean 

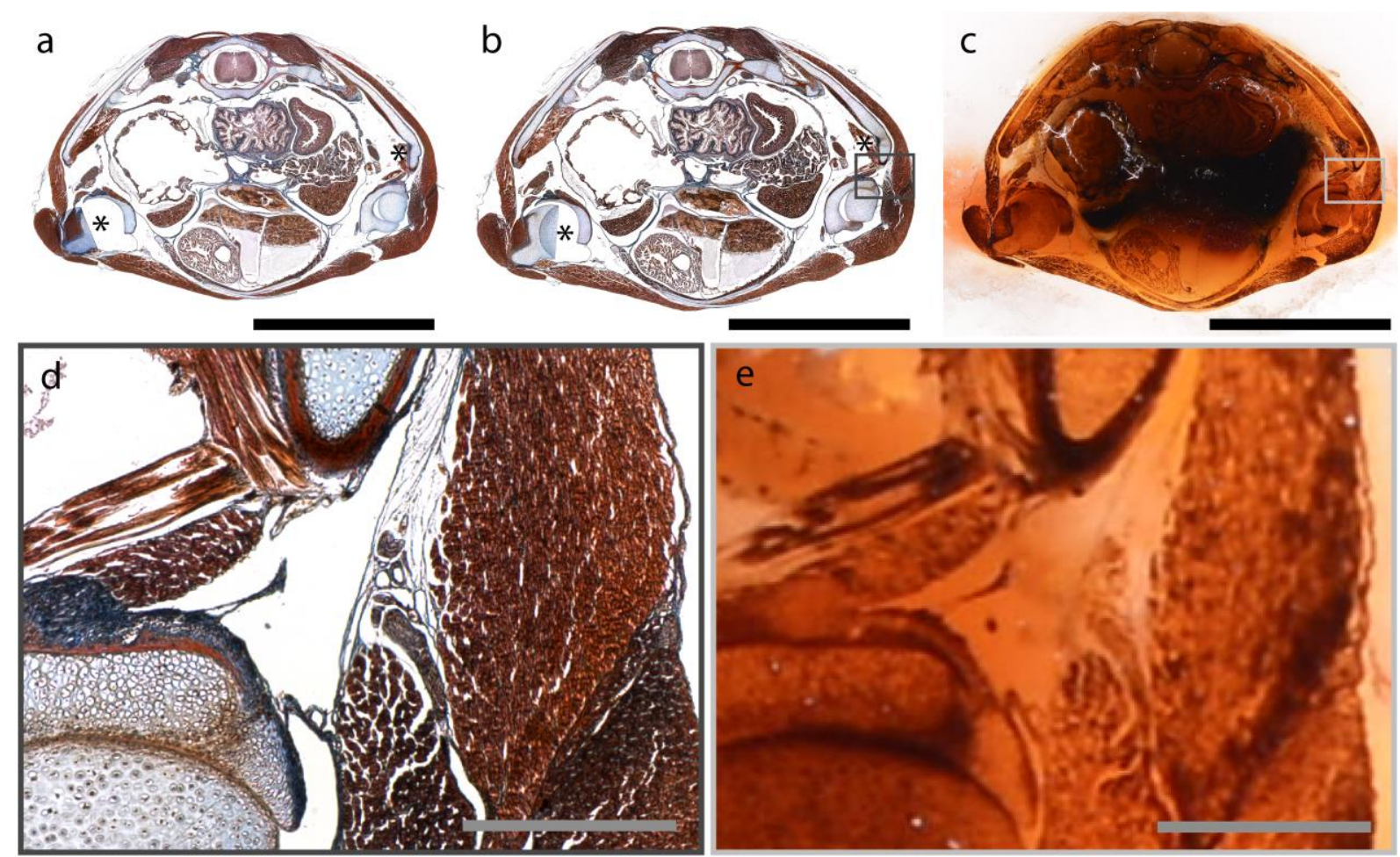

Fig. 7 Histological sections (a, b, d) and episcopic images (c, e) of Alytes obstetricans (ZMH

A12442). a: Digitized Azan stained histological section that was not contrasted with sodium sulfide. b: Digitized Azan stained histological section that was previously contrasted with sodium sulfide. Sections of $\mathrm{a}$ and $\mathrm{b}$ were located on the same glass slide and thus Azan-stained identically. c: Sodium sulfide contrasted episcopic image that shows the same section as in b. d: Detail of b showing different tissues and some brownish remains of the sodium sulfide staining. e: Detail of $\mathrm{c}$ showing the resolution limit of the episcopic images. *: regions damaged during sectioning, section mounting, or Azan-staining; black scales: $5 \mathrm{~mm}$; gray scales: $0.5 \mathrm{~mm}$ 


\section{Tables}

Table 1 Influence of focus plane to sensor distance, extension tube, and teleconverter on pixel size (first value) and field of view (second). Asterisk (*) denotes smallest pixel size (highest magnification) obtainable with respective equipment. Values were rounded to the nearest two decimals

\begin{tabular}{|l|l|l|l|}
\hline $\begin{array}{l}\text { focus plane to } \\
\text { sensor distance } \\
{[\mathrm{mm}]}\end{array}$ & Macro lens only & $\begin{array}{l}\text { Macro lens and } \\
\text { extension tube }\end{array}$ & $\begin{array}{l}\text { Macro lens and } \\
\text { teleconverter }\end{array}$ \\
\hline 310 & $\begin{array}{l}3.68 \mu \mathrm{m}^{*} \\
22.07 \times 14.71 \mathrm{~mm}^{2}\end{array}$ & - & - \\
\hline 320 & $\begin{array}{l}4.19 \mu \mathrm{m} \\
25.16 \times 16.78 \mathrm{~mm}^{2}\end{array}$ & - & - \\
\hline 324 & - & $2.53 \mu \mathrm{m}^{*}$ \\
& & $15.19 \times 10.13 \mathrm{~mm}^{2}$ & - \\
\hline 330 & $4.70 \mu \mathrm{m}$ & $2.97 \mu \mathrm{m}$ & - \\
& $28.21 \times 18.80 \mathrm{~mm}^{2}$ & $17.80 \times 11.87 \mathrm{~mm}^{2}$ & \\
\hline 334 & - & - & $2.63 \mu \mathrm{m}^{*}$ \\
& & & $15.77 \times 10.51 \mathrm{~mm}^{2}$ \\
\hline 340 & $5.19 \mu \mathrm{m}$ & $2.85 \mu \mathrm{m}$ \\
& $31.14 \times 20.76 \mathrm{~mm}^{2}$ & $22.79 \times 15.20 \mathrm{~mm}^{2}$ & $17.12 \times 11.41 \mathrm{~mm}^{2}$ \\
\hline 350 & $5.65 \mu \mathrm{m}$ & $4.23 \mu \mathrm{m}$ & $3.19 \mu \mathrm{m}$ \\
& $33.91 \times 22.60 \mathrm{~mm}^{2}$ & $25.36 \times 16.90 \mathrm{~mm}^{2}$ & $19.13 \times 12.76 \mathrm{~mm}^{2}$ \\
\hline 360 & $6.10 \mu \mathrm{m}$ & $4.78 \mu \mathrm{m}$ \\
& $36.57 \times 24.38 \mathrm{~mm}^{2}$ & $28.70 \times 19.14 \mathrm{~mm}^{2}$ & $3.54 \mu \mathrm{m}$ \\
& \multicolumn{2}{|c|}{$21.25 \times 14.16 \mathrm{~mm}^{2}$} \\
\hline
\end{tabular}




\section{Online Resources}

Online Resource 1 Protocol for paraffin embedding of Alytes obstetricans (ZMH A12442)

Online Resource 2 Scripts and documentation for Amira ${ }^{\circledR}$ macro MultiExport

Online Resource 3 Descriptive statistics of the sets of shortest distances between the observed midpoints and the respectively fitted lines

Online Resource 4 Results of Kruskal-Wallis rank sum test and Dunn's test on distance sets 